\title{
BMJ Open Systematic review and meta-analysis of prognostic factors for idiopathic inflammatory myopathy-associated interstitial lung disease
}

\author{
Hiroyuki Kamiya, ${ }^{1}$ Ogee Mer Panlaqui, ${ }^{2}$ Shinyu Izumi, ${ }^{3}$ Takashi Sozu ${ }^{4}$
}

To cite: Kamiya H, Panlaqui OM, Izumi S, et al. Systematic review and meta-analysis of prognostic factors for idiopathic inflammatory myopathy-associated interstitial lung disease. BMJ Open 2018;8:e023998. doi:10.1136/ bmjopen-2018-023998

- Prepublication history and additional material for this paper are available online. To view these files, please visit the journal online (http://dx.doi org/10.1136/bmjopen-2018023998).

Received 4 May 2018 Revised 25 August 2018 Accepted 17 October 2018

Check for updates

(C) Author(s) (or their employer(s)) 2018. Re-use permitted under CC BY-NC. No commercial re-use. See rights and permissions. Published by BMJ.

${ }^{1}$ School of Population and Global Health, University of Western Australia, Perth, Western Australia, Australia ${ }^{2}$ Department of Intensive Care Medicine, Northern Hospital, Epping, Victoria, Australia

${ }^{3}$ Department of Respiratory Medicine, National Center for Global Health and Medicine,

Tokyo, Japan

${ }^{4}$ Department of Information and Computer Technology, Faculty of Engineering, Tokyo University of Science, Tokyo, Japan

Correspondence to Dr Hiroyuki Kamiya; mlb04194@nifty.com

\section{ABSTRACT}

Objective To clarify prognostic factors for idiopathic inflammatory myopathy (IIM)-associated interstitial lung disease (ILD).

Design Systematic review and meta-analysis using the Grades of Recommendation, Assessment, Development and Evaluation system.

Data sources Medline, EMBASE and Science Citation Index Expanded were searched through 9 August 2018. Eligibility criteria for selecting studies The review includes primary studies addressing all-cause mortality of IIM-associated ILD. Potential prognostic factors were any clinical information related to the outcome.

Data extraction and synthesis Two reviewers extracted relevant data independently and assessed risk of bias using the Quality in Prognostic Studies tool. Meta-analysis was conducted using a random effects model and if inappropriate the results were reported qualitatively. Prognostic factors were determined based on statistically significant results derived from multivariate analysis.

Results Of a total of 5892 articles returned, 32 were deemed eligible for analysis and cumulatively, these studies reported 28 potential prognostic factors for all-cause mortality. Each study was subject to certain methodological constraints. The four prognostic factors, which demonstrated statistically significant results on both univariate and multivariate analyses, were as follows: age (MD 5.90, 3.17-8.63/HR 1.06, 1.02-1.10 and 2.31, 1.06-5.06), acute/subacute interstitial pneumonia (A/ SIP) (OR 4.85, 2.81-8.37/HR 4.23, 1.69-12.09 and 5.17, 1.94-13.49), percentage of predicted forced vital capacity (\%FVC) (OR 0.96, 0.95-0.98/HR 0.96, 0.93-0.99) and antiJo- 1 antibody (OR 0.35, 0.18-0.71/HR 0.004, 0.000030.54 ) (univariate/multivariate, $95 \% \mathrm{Cl}$ ). Other prognostic factors included ground glass opacity/attenuation (GGO/ GGA) and extent of radiological abnormality. The quality of the presented evidence was rated as either low or very low.

Conclusions Older age, A/SIP, lower value of $\% \mathrm{FVC}$, GGO/GGA and extent of radiological abnormality were demonstrated to predict poor prognosis for IIM-associated ILD while a positive test for anti-Jo- 1 antibody indicated better prognosis. However, given the weak evidence they should be interpreted with caution.

Trial registration number CRD42016036999.

\section{Strengths and limitations of this study}

- This systematic review and meta-analysis included primary research of multiple types to enable analysis of a larger cohort of patients with idiopathic inflammatory myopathy-associated interstitial lung disease that has previously been difficult in a single study due to disease rarity.

Due to the heterogeneity between studies and the potential risk of bias in the reviewed articles, the interpretation and application of these findings is potentially constrained.

- Determination of prognostic factors may have been affected by a small number of studies that conducted multivariate analysis.

\section{BACKGROUND}

Polymyositis, dermatomyositis, clinically amyopathic dermatomyositis (CADM) and antisynthetase syndrome (ASS) are all categorised into idiopathic inflammatory myopathy (IIM). ${ }^{1}$ Irrespective of their diverse clinical manifestations, they are regarded as being in the same disease spectrum. These diseases are characterised by inflammatory myositis, unique cutaneous findings or the presence of anti-aminoacyl-transfer RNA synthetase (ARS) antibody in the blood. ${ }^{2}$ Interstitial lung disease (ILD) is another well-recognised complication of IIM and worsens the prognosis of the disease. ${ }^{3}$ However, there is substantial variation in the progression of disease among patients with IIM-associated ILD. ${ }^{4}$ Although some studies report potential prognostic factors for IIM-associated ILD, all studies had a small sample size, thus prognostic factors seem to be disparate and anecdotal. ${ }^{5}$ Due to the rarity of the disease, it is likely that a large cohort study to address this clinical question is not feasible. Therefore, this systematic review and meta-analysis was designed to clarify prognostic factors for IIM-associated ILD, with the view of guiding 
all parties concerned with this complicated spectrum of diseases. This study was registered at PROSPERO (CRD42016036999).

\section{METHODS}

This review was conducted and reported according to the Preferred Reporting Items for Systematic Reviews and Meta-Analyses ${ }^{6}$ and the Meta-analysis of Observational Studies in Epidemiology statement. ${ }^{7}$ The methods underpinning this review are only briefly described as it has been covered in-depth in a separate protocol paper. ${ }^{8}$ Some revisions and additions were necessary after project commencement. The requirement of follow-up periods of at least 6 months was removed to include some patients who demonstrate a drastic course. Therapeutic intervention was excluded from potential prognostic factors as the effect of treatment on prognosis will be confounded by a number of factors and difficult to be accurately evaluated in prognostic studies. In addition, potential prognostic factors were defined as the ones reported in at least five studies as it would enhance the applicability of the findings. Finally, the outcome was focused on all-cause mortality considering its most clinical relevance.

\section{Eligibility}

Journal articles and reports of other types examining ILD associated with polymyositis, dermatomyositis, CADM and ASS at adult onset ( $>16$ years of age) were included in this systematic review and meta-analysis. Polymyositis, dermatomyositis and CADM were diagnosed based on the criteria set out by Bohan and Peter ${ }^{9} 10$ and Sontheimer. ${ }^{11}$ However, we removed the requirement set out by those researchers necessitating at least 6 months' patient follow-up. This decision was made to capture all potential patients, including those who may have died over a short period of time. ${ }^{12}$ ASS was included if a complication of ILD was noted in addition to a positive test for anti-ARS antibody and involvement of another organ, such as myopathies and unique cutaneous manifestations. The diagnosis of ILD was made based on physical exams, pulmonary function tests and radiological abnormalities. Juvenile myositis and overlap myositis were excluded from the review. Potential prognostic factors included any clinical information related to the outcome of interest. However, therapeutic intervention was excluded and we only focused on factors reported in at least five studies. The primary outcome of interest was all-cause mortality. Studies eligible for review included primary studies of any type (excluding case reports or case series), presented as full texts written in English. Conference proceedings and reports only showing abstracts were excluded.

\section{Search of studies}

Two reviewers (HK and OMP) independently searched the electronic databases of Medline (Ovid) (from inception through October 2016), EMBASE (Ovid) (from inception through October 2016), Science Citation Index
Expanded (Web of Science) (from inception through December 2016) and Google Scholar (18 December 2016). The search was further updated on 9 August 2018. Subject headings and text words relating to study populations formed a string of search terms. These included terms such as polymyositis, dermatomyositis and interstitial lung disease and their synonyms combined with the methodology filter of 'prognosis', which was modified according to each database (online supplementary e-Appendix). Reference lists from eligible studies and relevant review articles were also hand-searched and experts were contacted for additional reports.

\section{Selection of studies and data extraction}

Two reviewers (HK and OMP) independently selected eligible studies examining titles and abstracts of all the retrieved articles, referring to their full texts if needed. Data were extracted by the same reviewers based on a predefined data extraction form. This form included first author name, year of publication, study location, study design, length of follow-up, pattern of ILD, study population, and demographic characteristics of participants, presence of autoantibodies, clinical outcomes, potential prognostic factors, methods of statistical analysis, summary statistics and items associated with risk of bias. Both unadjusted and adjusted results were drawn and adjusted factors were also extracted if available. Any disagreement between the reviewers on the articles to be included was resolved through discussion. If the same clinical information was mentioned in five or more studies, we assumed clinical relevance.

\section{Assessment of risk of bias}

Risk of bias in individual studies was assessed using the Quality in Prognostic Studies tool. ${ }^{13}$ This tool consists of six domains, which culminate to evaluate an overall risk of bias. Based on this tool, risk of bias is rated as either high, moderate or low. A study satisfying low risk of bias in all six domains was designated as a study with an overall low risk of bias.

\section{Measurement of the association}

Measuring the strength of association of predefined outcomes with potential prognostic factors was determined using odds ratios (ORs), risk ratios (RRs) and hazard ratios (HRs). The mean difference (MD) or the standardised MD was considered an appropriate measure when potential prognostic factors were continuous variables. If certain prognostic factors were reported in multiple studies from the same research group, the result with the largest sample size was prioritised in the analysis. This meant that other studies by that group might still be included because potential prognostic factors reported were diverse between studies even if they were conducted by the same research group.

\section{Data synthesis}

The effect of each potential prognostic factor was combined if it could be summarised with the same 
statistics in three or more studies. Rapidly progressive and Hamman-Rich-like pattern of ILD was classified together with acute/subacute interstitial pneumonia (A/ $\mathrm{SIP}$ ) as such designation has been previously reported in the literature. ${ }^{14}$ The results generated from univariate analysis were combined separately from those generated using multivariate analysis. In both cases, random effects models with the inverse variance method were used. All analyses were undertaken using the statistical software Review Manager (RevMan) V.5.3 (Copenhagen: The Nordic Cochrane Centre, The Cochrane Collaboration, 2014). The $95 \%$ prediction interval (PI) was presented if heterogeneity between studies was identified for potential prognostic factors that demonstrated statistically significant results. ${ }^{15}$

\section{Heterogeneity}

Heterogeneity was assessed by Cochran's $Q$ statistics and the $\mathrm{I}^{2}$. Statistical significance was set at $\mathrm{p}$ value $\leq 0.1$ with the magnitude of heterogeneity subdivided into four categories. These categories were designated as: not important $(0 \%-30 \%)$, moderate $(30 \%-50 \%)$, substantial $(50 \%-70 \%)$ and considerable $(70 \%-100 \%) .{ }^{16}$ If heterogeneity between studies was suspected, subgroup analysis was to be conducted. Subgroup analysis was centred on the potential causes of heterogeneity between studies. Suspected causes of heterogeneity included: varying classifications of disease and different types of ILD under observation. Furthermore, sensitivity analysis was to be conducted on studies deemed to have low risk of bias.

\section{Metabiases}

Small study bias such as publication bias was evaluated using Egger's test and depicted using a funnel plot to demonstrate asymmetry. They were only conducted on meta-analyses featuring 10 or more studies. ${ }^{17}$ Statistical significance for asymmetry was set at $p$ value $\leq 0.1$.

\section{Determining prognostic factors}

If a potential prognostic factor demonstrated a statistically significant result in univariate analysis using pooled data in the meta-analysis and for non-pooled data the effect was in the same direction in the majority of the studies $(\geq 75 \%)$, it was deemed to be a prognostic factor. The result of multivariate analysis was interpreted in the same way. A final decision of determining prognostic factors was made based on both significant and consistent results of multivariate analysis in the majority of the studies $(\geq 75 \%)$.

\section{Evaluating the presented evidence}

The Grades of Recommendation, Assessment, Development and Evaluation (GRADE) system was applied to evaluate the evidence of identified prognostic factors. ${ }^{18}$

\section{Patient and public involvement}

There was no patient and public involvement in the whole process of conducting this research.
RESULTS

\section{Search strategy}

A total of 5892 articles were identified using four separate electronic databases. After excluding 1115 duplicates from these, 1226 were removed as they did not relate to the topic of interest. A further 3040 were removed due to ineligible study type. From the remaining studies, 402 did not meet the inclusion criteria. Of the remaining 109 articles, which were obtained as full texts, 77 met the eligibility criteria for inclusion. No additional reports were identified from other potential sources (online supplementary e-Figure 1).

\section{Potential prognostic factors}

A total of 28 potential prognostic factors were identified for all-cause mortality from 32 of the 77 total eligible studies (online supplementary e-Table 1 ). The remaining 45 studies were excluded for further analysis as they did not identify potential prognostic factors that featured in five or more studies.

\section{Overview of included studies}

A total of 30 of the 32 studies were conducted in Asia. The majority of studies took place in Japan (22/32), followed by China (6/32) and Korea (2/32). The remaining two studies were conducted in the USA and Mexico, respectively. Twenty-one studies and one study used a retrospective and a prospective cohort study design, respectively, with the remaining using a case-control design. Twenty-five studies had a sample size of $\leq 50$ people, four had 51-100 people and three had $>100$ people ${ }^{19-50}$ (online supplementary e-Table 2). Considering the largest sample size among multiple studies conducted by the same research group (Hozumi et al, ${ }^{19}$ Enomoto et al, ${ }^{20}$ Hozumi $e t a l^{21}$ and Fujisawa $e t a l^{22}$; Gono et $a l^{23}$ and Gono et $a l^{24} ;$ Li $e t a l^{25}$, Zou $e t a l^{26}$ and Ye $e t a l^{27}$ ), a total of 962 participants were included in the analysis of potential prognostic factors for all-cause mortality (online supplementary e-Table 2).

\section{Risk of bias}

In many studies the process of selecting participants was unclear. As a result, their risk of bias was high. Furthermore, the rate of attrition in most cohort studies was not explicitly stated, which could have further biased results. There was high risk of bias for the categories of confounding, statistical analysis and reporting in all studies. This was judged on the basis that many potential confounders were not addressed and insufficient detail was provided when describing the models used for analysis. Consequently, all studies were noted to have some methodological flaw (online supplementary e-Table 3).

\section{Statistical analysis}

The effects of all 28 potential prognostic factors for all-cause mortality were reported by univariate analysis. Multivariate analysis was conducted for 22 potential prognostic factors. Only one potential prognostic factor was pooled for multivariate analysis, while the results of 


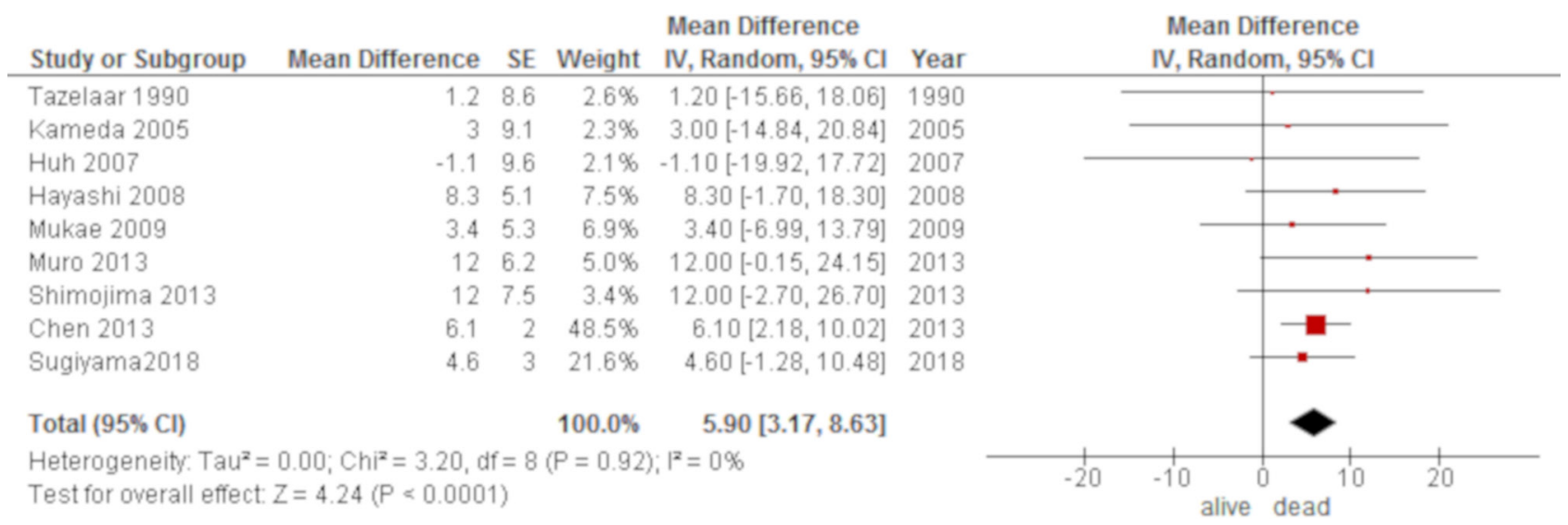

Figure 1 Forest plot of the result of univariate analysis for age. Nine studies were pooled for meta-analysis and a total of 267 patients with 22 of polymyositis, 126 of dermatomyositis, 93 of clinically amyopathic dermatomyositis (CADM) and 26 of polymyositis or dermatomyositis were included. Non-survivors were significantly older than survivors with the mean difference $(\mathrm{MD})$ of 5.90 years $(95 \% \mathrm{Cl} 3.17$ to $8.63, p<0.0001)$ and there was no heterogeneity $\left(X^{2}=3.20, p=0.92, I^{2}=0 \%\right)$.

univariate analysis were combined for 21 potential prognostic factors (online supplementary e-Tables 4 and 5 ).

Univariate analysis

The result of univariate analysis identified 13 potential prognostic factors that were statistically significant for pooled data in the meta-analysis, and for non-pooled studies the effect was in the same direction in the majority of the studies. These potential prognostic factors included age, dermatomyositis, A/SIP, fever, percentage of predicted forced vital capacity (\%FVC), reticular opacity, anti-Jo-1 antibody, anti-melanoma differentiation antigen 5 antibody (anti-MDA5 antibody), ferritin, alveolar-arterial oxygen difference $\left(\mathrm{A}-\mathrm{aDO}_{2}\right)$, arterial oxygen pressure $\left(\mathrm{PaO}_{2}\right)$, C-reactive protein (CRP) and aspartate aminotransferase (AST) (online supplementary e-Table 4, figures 1-4 and online supplementary e-Figures 2-5).
Multivariate analysis and identifying prognostic factors

Among the 13 factors identified through univariate analysis, multivariate result was not available for reticular opacity. In addition, dermatomyositis, A- $\mathrm{aDO}_{2}, \mathrm{PaO}_{2}$ and AST were statistically non-significant on multivariate analyses although they were all based on the result of a single study. The results of multivariate analysis for anti-MDA5 antibody, fever and CRP were reported in a few studies and only one study demonstrated a significant result for all these factors. The combined result of multivariate analyses for ferritin was also statistically non-significant (online supplementary e-Table 5 and e-Figure 6). The remaining four potential prognostic factors were all significant on multivariate analyses and thus considered to satisfy the criteria for legitimate prognostic factors although they were based on the result of one or two studies. Similarly significant were the other two factors

\begin{tabular}{|c|c|c|c|c|c|}
\hline Study or Subgroup & log[Odds Ratio] & SE & Weight & $\begin{array}{c}\text { Odds Ratio } \\
\text { IV, Random, } 95 \% \mathrm{Cl}\end{array}$ & Year \\
\hline Kang 2005 & 2.98 & 0.55 & $11.9 \%$ & $19.69[6.70,57.86]$ & 2005 \\
\hline Huh 2007 & 1.16 & 0.43 & $14.5 \%$ & $3.19[1.37,7.41]$ & 2007 \\
\hline Mukae 2009 & 1.93 & 1.36 & $3.5 \%$ & $6.89[0.48,99.04]$ & 2009 \\
\hline Gono-Satoh 2010 & 1.61 & 1.38 & $3.4 \%$ & $5.00[0.33,74.79]$ & 2010 \\
\hline Su-yun 2010 & 2.05 & 0.39 & $15.5 \%$ & $7.77[3.62,16.68]$ & 2010 \\
\hline Sun 2013 & 2.19 & 0.99 & $5.8 \%$ & $8.94[1.28,62.20]$ & 2013 \\
\hline Muro 2013 & 1.73 & 1.55 & $2.8 \%$ & $5.64[0.27,117.68]$ & 2013 \\
\hline Fujisawa 2014 & 1.7 & 0.47 & $13.6 \%$ & $5.47[2.18,13.75]$ & 2014 \\
\hline Zou 2015 & 0.73 & 0.6 & $10.9 \%$ & $2.08[0.64,6.73]$ & 2015 \\
\hline Zhang2018 & 0.71 & 0.29 & $17.9 \%$ & $2.03[1.15,3.59]$ & 2018 \\
\hline Total $(95 \% \mathrm{CI})$ & & & $100.0 \%$ & $4.85[2.81,8.37]$ & \\
\hline
\end{tabular}

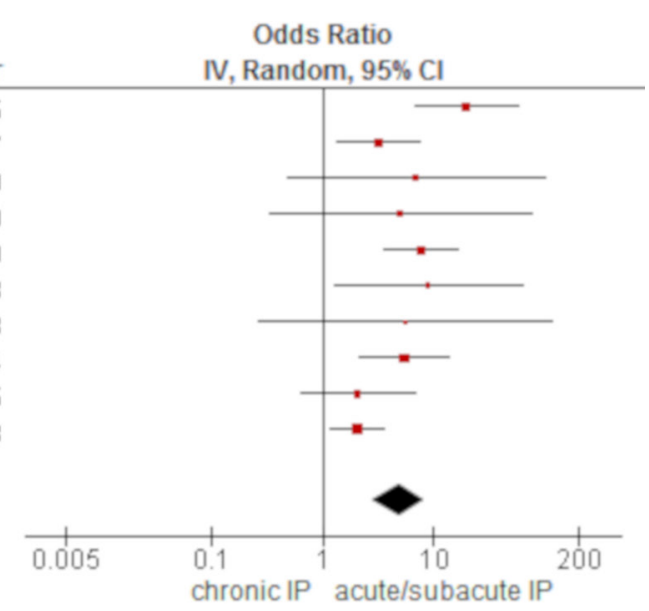

Figure 2 Forest plot of the result of univariate analysis for acute/subacute interstitial pneumonia (A/SIP). Ten studies were pooled for meta-analysis and a total of 460 patients with 48 of polymyositis, 223 of dermatomyositis, 156 of clinically amyopathic dermatomyositis (CADM) and 33 of polymyositis or dermatomyositis were included. There was a significant difference of all-cause mortality between A/SIP and chronic IP with the OR of $4.85(95 \% \mathrm{Cl} 2.81$ to $8.37, \mathrm{p}<0.00001)$. A point estimate of individual studies was all in the same direction although substantial heterogeneity was identified $\left(X^{2}=19.50, p=0.02\right.$, $\left.\mathrm{I}^{2}=54 \%\right)$. The $95 \%$ prediction interval was between 0.80 and 29.40 . 


\begin{tabular}{|c|c|c|c|c|c|c|c|c|c|}
\hline Study or Subgroup & log[Odds Ratio] & SE & Weight & $\begin{array}{c}\text { Odds Ratio } \\
\text { IV, Random, } 95 \% \mathrm{Cl}\end{array}$ & Year & \multicolumn{4}{|c|}{$\begin{array}{c}\text { Odds Ratio } \\
\text { IV, Random, } 95 \% \mathrm{Cl}\end{array}$} \\
\hline Tanizawa 2013 & -0.01 & 0.02 & $18.3 \%$ & $0.99[0.95,1.03]$ & 2013 & & $\longrightarrow$ & $H$ & \\
\hline Fujisawa 2014 & -0.04 & 0.01 & $65.0 \%$ & $0.96[0.94,0.98]$ & 2014 & & & & \\
\hline Zou 2015 & -0.04 & 0.03 & $8.4 \%$ & $0.96[0.91,1.02]$ & 2015 & & & - & \\
\hline Rojas-Serrano 2015 & -0.07 & 0.03 & $8.4 \%$ & $0.93[0.88,0.99]$ & 2015 & & & & \\
\hline Total $(95 \% \mathrm{Cl})$ & & & $100.0 \%$ & $0.96[0.95,0.98]$ & & & & & \\
\hline $\begin{array}{l}\text { Heterogeneity: } \mathrm{Tau}^{2}= \\
\text { Test for overall effect: }\end{array}$ & $\begin{array}{l}.00 ; \mathrm{Chi}^{2}=3.13, \mathrm{~d} \\
=4.23(P \approx 0.000\end{array}$ & $f=3(t$ & $=0.37)$ & $I^{2}=4 \%$ & & 0.85 & 0.9 & 1.1 & 1.2 \\
\hline
\end{tabular}

Figure 3 Forest plot of the result of univariate analysis for forced vital capacity of predicted (\%FVC). Four studies were pooled for meta-analysis and a total of 245 patients with 41 of polymyositis, 63 of dermatomyositis, 98 of clinically amyopathic dermatomyositis (CADM) and 43 of antisynthetase syndrome (ASS) were included. \%FVC was significantly associated with all-cause mortality with the OR of $0.96(95 \% \mathrm{Cl} 0.95$ to $0.98, \mathrm{p}<0.0001)$. A point estimate of individual studies was all in the same direction although not important heterogeneity was identified $\left(X^{2}=3.13, p=0.37, I^{2}=4 \%\right)$. The $95 \%$ prediction interval was between 0.92 and 0.99 .

(ground glass opacity/attenuation (GGO/GGA) and extent of abnormality on high resolution CT (HRCT)) (online supplementary e-Table 5) although by univariate analysis GGO/GGA demonstrated a non-significant combined effect (online supplementary e-Figure 7) while extent of abnormality showed inconsistent point estimates between studies (online supplementary e-Table 4).

The remaining 13 potential prognostic factors had non-significant results by meta-analysis and where data were not combined, these factors demonstrated inconsistent results between studies through univariate analysis. The result of multivariate analyses was unavailable or non-significant for all these factors. Therefore, these potential prognostic factors were considered less likely to be associated with all-cause mortality (online supplementary e-Tables 4 and 5 and e-Figures 8-19).

In summary, six prognostic factors were identified, of which four (age, A/SIP, \%FVC and anti-Jo-1 antibody) met the criteria for both univariate and multivariate analysis. The remaining two factors (GGO/GGA and extent of abnormality) were identified based solely on the result of multivariate analysis. However, multivariate results for all these prognostic factors were derived from only one or two studies (online supplementary e-Table 5).

\section{Effect of prognostic factors}

Based on the results of univariate analysis, non-survivors were significantly older than survivors with a MD of 5.90 (95\% CI 3.17 to 8.63 ) (figure 1 ). Of the remaining five studies excluded from meta-analysis, four also revealed a similar trend. A multivariate analysis reported by two studies demonstrated a significant result with HRs of 1.06 (95\% CI 1.02 to 1.10 ) and 2.31 (95\% CI 1.06 to 5.06 ), respectively (online supplementary e-Table 5).

Univariate analysis determined that A/SIP was significantly associated with all-cause mortality when compared with chronic IP with an OR of 4.85 (95\% CI 2.81 to 8.37) (figure 2). Although the $95 \%$ PI ranging from 0.80 to 29.40 suggested an inconclusive association, two studies which conducted a multivariate analysis examining A/ SIP reported a significant result with HRs of 4.23 (95\% CI 1.69 to 12.09$)$ and 5.17 (95\% CI 1.94 to 13.49$)$, respectively (online supplementary e-Table 5 ).

Univariate analysis determined that $\% \mathrm{FVC}$ was significantly associated with all-cause mortality, with an OR of 0.96 (95\% CI 0.95 to 0.98$)$ (figure 3$)$. The $95 \%$ PI ranging from 0.92 to 0.99 also supported this significant result. Similarly, a multivariate analysis reported by one study also demonstrated a significant association with a HR of

\begin{tabular}{|c|c|c|c|c|c|c|c|c|c|}
\hline Study or Subgroup & log[Odds Ratio] & SE & Weight & $\begin{array}{l}\text { Odds Ratio } \\
\text { IV, Random, } 95 \% \mathrm{Cl}\end{array}$ & Year & \multicolumn{4}{|c|}{$\begin{array}{c}\text { Odds Ratio } \\
\text { IV, Random, } 95 \% \mathrm{Cl}\end{array}$} \\
\hline Ito 1999 & -1.5 & 1.36 & $6.7 \%$ & $0.22[0.02,3.21]$ & 1999 & & & & \\
\hline Mukae 2009 & -1.22 & 1.39 & $6.4 \%$ & $0.30[0.02,4.50]$ & 2009 & & & & \\
\hline Fujisawa 2014 & -0.51 & 0.55 & $41.1 \%$ & $0.60[0.20,1.76]$ & 2014 & & - & 一 & \\
\hline Rojas-Serrano 2015 & -1.97 & 0.8 & $19.4 \%$ & $0.14[0.03,0.67]$ & 2015 & & & & \\
\hline Sugiyama2018 & -1.08 & 1.01 & $12.2 \%$ & $0.34[0.05,2.46]$ & 2018 & & & & \\
\hline Zhang2018 & -0.97 & 0.94 & $14.1 \%$ & $0.38[0.06,2.39]$ & 2018 & & & & \\
\hline Total $(95 \% \mathrm{Cl})$ & & & $100.0 \%$ & $0.35[0.18,0.71]$ & & & & & \\
\hline \multicolumn{6}{|c|}{$\begin{array}{l}\text { Heterogeneity: } \operatorname{Tau}^{2}=0.00 ; \mathrm{Chi}^{2}=2.42, \mathrm{df}=5(\mathrm{P}=0.79) ;\left.\right|^{2}=0 \% \\
\text { Test for overall effect: } Z=2.95(P=0.003)\end{array}$} & 0.005 & 0.1 & $\begin{array}{c}10 \\
\text { negative }\end{array}$ & 200 \\
\hline
\end{tabular}

Figure 4 Forest plot of the result of univariate analysis for anti-Jo-1 antibody. Six studies were pooled for meta-analysis and a total of 403 patients with 30 of polymyositis, 162 of dermatomyositis, 54 of clinically amyopathic dermatomyositis (CADM), 43 of antisynthetase syndrome (ASS) and 114 of polymyositis, dermatomyositis and CADM were included. Anti-Jo-1 antibody was significantly protective against all-cause mortality with the OR of 0.35 ( $95 \% \mathrm{Cl} 0.18$ to $0.71, p=0.003)$. There was no heterogeneity $\left(\mathrm{X}^{2}=2.42, \mathrm{p}=0.79, \mathrm{I}^{2}=0 \%\right)$. 
0.96 (95\% CI 0.93 to 0.99 ) (online supplementary e-Table 5).

Anti-Jo-1 antibody was shown to be protective against all-cause mortality through univariate analysis, with an OR of 0.35 (95\% CI 0.18 to 0.71 ) (figure 4). One study using multivariate analysis demonstrated a significant protective effect for anti-Jo- 1 antibody, with a HR of 0.004 (95\% CI 0.00003 to 0.54 ) (online supplementary e-Table $5)$.

A positive GGO/GGA on HRCT was not significantly associated with all-cause mortality with an OR of 1.51 (95\% CI 0.88 to 2.60 ) shown by pooled data of univariate analysis (online supplementary e-Figure 6). However, one study excluded from meta-analysis reported a significant result for GGO/GGA, with a HR of 5.87 (95\% CI 1.43 to 24.01$)$. In contrast, a multivariate analysis reported by another study demonstrated a significant result with a HR of 7.68 (95\% CI 1.37 to 43.03 ) (online supplementary e-Table 5).

The result of extent of abnormality on HRCT was inconsistent between studies, as shown by univariate analysis. Two studies demonstrated a significant association of extent of abnormality with all-cause mortality (HR 1.15 (95\% CI 1.05 to 1.27) and MD 8.49 (95\% CI 2.72 to 14.26), respectively) whereas the other two studies reported non-significant point estimates in the opposite direction (HR 0.99 (95\% CI 0.95 to 1.02 ) and median $231.4 \%$ vs $247.8 \%$, respectively) (online supplementary e-Table 4). In contrast, a multivariate analysis reported by one study demonstrated a significant result with a HR of 1.13 (95\% CI 1.01 to 1.28) (online supplementary e-Table $5)$.

\section{Additional analyses}

Subgroup analysis was not undertaken due to the limited availability of demographic descriptions in the included studies. Additionally, due to the poor bias rating in most of the included studies, sensitivity analysis was deemed superfluous. Small study bias was evaluated for the analysis of sex and A/SIP as only these two factors were reported in at least 10 studies. A funnel plot was symmetrical and Egger's test demonstrated non-significant results for both factors ( $\mathrm{p}=0.34$ and 0.31 , respectively) (online supplementary e-Figures 20 and 21), which indicated that there was no apparent small study bias.

\section{Quality of evidence}

The GRADE system rated the quality of evidence for identified prognostic factors as either low or very low (online supplementary e-Table 6).

\section{DISCUSSION}

We demonstrated that age, A/SIP, \%FVC, anti-Jo-1 antibody, GGO/GGA and extent of abnormality on HRCT were associated with a prognosis for IIM-associated ILD. To the best of our knowledge, this is the first systematic review and meta-analysis addressing prognostic factors of this disease group. Due to the difficulty in conducting large-scale research for this rare disease, this review is the foremost evidence assessing this area of research. This review retrieved and investigated all clinical information relevant and commonly used in clinical practice for the assessment of IIM-associated ILD. As the review was previously reported and registered as a protocol paper, its methodology is sound.

All prognostic factors identified in this review seem to have important clinical implications. One of the greatest factors affecting the prognosis of this disease is age. This generally occurs as ageing reduces tolerance to disease and often leads to disease comorbidity. For example, hypertension is a common disease for elderly people. If a patient with IIM-associated ILD also suffers from heart failure or chronic kidney disease caused by hypertension, treatment will be more challenging and all-cause mortality will eventually increase. Both \% FVC (indicating the capacity of pulmonary function) and extent of abnormality on HRCT reflect the damage that this disease causes to lung parenchyma. Additionally, the radiological finding of GGO/GGA may imply resistance to treatment of the disease. ${ }^{51}$ Autoantibodies such as anti-Jo-1 antibody and anti-MDA5 antibody are noted as important factors that define certain disease phenotype across the spectrum of these conditions. Anti-Jo-1 antibody may be associated with chronic IP, ${ }^{52}$ while anti-MDA5 antibody is more likely associated with onset of acute IP, ${ }^{23} 263538$ which may lead to a difference in prognosis for the disease. Although anti-MDA5 antibody was noted to be statistically significant using pooled data through univariate analysis, only one study demonstrated a significant result on multivariate analysis, which was also true for fever and CRP. Similarly, other factors such as dermatomyositis, ferritin, $\mathrm{A}-\mathrm{aDO}_{2}$ and $\mathrm{PaO}_{2}$ demonstrated statistically non-significant results through multivariate analysis although they were all significant under univariate analysis. However, some of these factors may still be related to all-cause mortality, particularly the presence of anti-MDA5 antibody may possibly predict the development of rapidly progressive and intractable ILD, which can also cause fever and elevated value of CRP as a consequence of extensive pulmonary inflammation. ${ }^{23} 263538$ It should be noted that the results of multivariate analysis were primarily based on only one or two studies, which attenuates the strength of some of the presented evidence. As a result, the interpretation of these findings warrants attention and should be confirmed by future studies.

There is also another point that may affect the findings of this review. It has been shown that anti-ARS antibody and anti-MDA5 antibody strongly predict a complication of ILD. ${ }^{53} 54$ The key difference between the two antibodies is that anti-ARS antibody can be identified in overlap myositis ${ }^{53}$ while anti-MDA5 antibody is specific to CADM or dermatomyositis. ${ }^{54}$ It was observed that anti-MDA5 antibody is more frequently reported for Japanese patients. ${ }^{55}$ By excluding components of other connective tissue diseases, this review may have contained 
an unbalanced selection of Japanese studies with a focus on anti-MDA5 antibody. Consequently, the findings of this review may be strongly influenced by clinical features of Japanese patients, who often present with a fatal form of ILD. ${ }^{23} 3538$ It has been observed that more severe forms of ILD are closely associated with anti-MDA5 antibody, ${ }^{23} 3538$ with other evidence suggesting a strong environmental and genetic link as likely causes. ${ }^{56}{ }^{57}$ Contrary to most studies, one study in a Western population reported anti-MDA5 antibody in a different clinical manifestation of the disease. ${ }^{58}$ Due to these discrepancies, the presented evidence may not be applicable outside of an Asian population. Further investigation is needed to clarify this issue.

Although this review identified the key prognostic factors for IIM-associated ILD, we acknowledge that clinicians are likely more interested in the clinical impact of each prognostic factor rather than their enumeration. However, this study was not intended to rank prognostic factors or suggest which are more important in the clinical setting. Our findings were simply based on the summary results of different studies, with diverse statistics used to estimate the effect size of each prognostic factor. As a result, it was considered inappropriate to compare the impact of each prognostic factor identified in this review. Furthermore, the results of continuous data such as age and \%FVC seem to lack profound clinical implications and may be regarded as less meaningful as it is difficult for clinicians to apply these findings in a practical setting. However, this was caused by the constraints of the data and handling continuous variables as such is also noted to be an appropriate statistical approach. ${ }^{59}$

This review, while comprehensive in its approach, has some methodological shortcomings. First, all evidence obtained for this review was rated as either low or very low quality by the GRADE system. ${ }^{18}$ This was typically a result of the phase of included studies as prognostic research, inherent methodological limitations of each study and publication bias. As this review was designated as a phase 1 study, the level of evidence started at moderate and was downgraded to low due to publication bias (a common feature in prognostic research). Additionally, all prognostic factors are subject to limitation as all included studies had some risk of bias. This may be a result of the small size of the included studies or the interconnectedness of a small number of research groups/medical institutions. Second, we selected polymyositis, dermatomyositis, CADM and ASS to represent all patients with IIM-associated ILD. This amalgamation of diseases may have contributed to the observed heterogeneity between studies. Although we planned to conduct subgroup analysis to identify potential sources of heterogeneity, it was difficult due to the limited availability of data to make a stratification with common demographic characteristics. Consequently, it is unclear whether all prognostic factors identified in this review will universally affect the prognosis of all disease subsets across this disease spectrum. Lastly, we decided to focus on potential prognostic factors reported in five or more studies. Our submitted protocol paper omitted this detail. ${ }^{8}$ Specifying this criterion may have helped identify those prognostic factors most commonly used in clinical practice and will thus improve the applicability of our findings. The decision to include patients with less than 6 months' follow-up may have also benefitted the reporting of prognostic factors for all subsets of this disease. Regardless of these limitations we believe this systematic review and meta-analysis can be used as a valuable guide to IIM-associated ILD. However, the review needs to be updated with additional future reports and address other potential prognostic factors and outcomes, which were not put forward in this research.

\section{CONCLUSIONS}

Key prognostic factors for IIM-associated ILD were identified from the available literature. Older age, A/SIP, lower value of \% FVC, and GGO/GGA and extent of abnormality on HRCT were demonstrated to predict poor prognosis while the presence of anti-Jo-1 antibody indicated better prognosis for the disease. However, interpreting and applying these findings must be considered in light of poor quality of evidence, in particular the methodological shortcomings representing the studies constituting this review.

Acknowledgements We thank Istvan T Kabdebo at School of Population and Global Health, University of Western Australia, for his support of editing the manuscript.

Contributors HK planned the entire research project and analysed the data. He also summarised the result and wrote the manuscript. HK has full access to the data and takes responsibility for its integrity as well as the accuracy of the analysis. OMP contributed to the design of the research project and conducted the literature search and data extraction. He was also involved in revising the manuscript. SI contributed to the design of the research project, in particular, selecting the appropriate sample population. TS contributed to the planning of statistical analysis, in particular, determining the appropriate statistical methods to report summary effects and data synthesis. All researchers provided thoughts and opinions to compile a draft paper with revisions and then approved the final version of the manuscript.

Funding The authors have not declared a specific grant for this research from any funding agency in the public, commercial or not-for-profit sectors.

Competing interests None declared.

Patient consent Not required.

Provenance and peer review Not commissioned; externally peer reviewed.

Data sharing statement The data set used and/or analysed for this review will be available from the corresponding author on a reasonable request and may become open to the public through a digital repository (such as Dryad) after the final result is published in a journal.

Open access This is an open access article distributed in accordance with the Creative Commons Attribution Non Commercial (CC BY-NC 4.0) license, which permits others to distribute, remix, adapt, build upon this work non-commercially, and license their derivative works on different terms, provided the original work is properly cited, appropriate credit is given, any changes made indicated, and the use is non-commercial. See: http://creativecommons.org/licenses/by-nc/4.0/.

\section{REFERENCES}

1. Gerami P, Schope JM, McDonald L, et al. A systematic review of adult-onset clinically amyopathic dermatomyositis 
(dermatomyositis siné myositis): a missing link within the spectrum of the idiopathic inflammatory myopathies. J Am Acad Dermatol 2006;54:597-613.

2. Hervier B, Benveniste O. Clinical heterogeneity and outcomes of antisynthetase syndrome. Curr Rheumatol Rep 2013;15:349.

3. Hallowell RW, Danoff SK. Interstitial lung disease associated with the idiopathic inflammatory myopathies and the antisynthetase syndrome: recent advances. Curr Opin Rheumatol 2014;26:684-9.

4. Kiely PDW, Chua F. Interstitial lung disease in inflammatory myopathies: clinical phenotypes and prognosis. Curr Rheumatol Rep 2013;15:1-7.

5. Selva-O'Callaghan A, Labrador-Horrillo M, Muñoz-Gall X, et al. Polymyositis/dermatomyositis-associated lung disease: analysis of a series of 81 patients. Lupus 2005;14:534-42.

6. Moher D, Liberati A, Tetzlaff J, et al. Preferred reporting items for systematic reviews and meta-analyses: the PRISMA statement. Ann Intern Med 2009;151:264-9.

7. Stroup DF, Berlin JA, Morton SC, et al. Meta-analysis of observational studies in epidemiology: a proposal for reporting. Meta-analysis Of Observational Studies in Epidemiology (MOOSE) group. JAMA 2000;283:2008-12.

8. Kamiya H, Panlaqui OM, Izumi S, et al. Prognostic factors of idiopathic inflammatory myopathies complicated with interstitial lung disease: protocol for a systematic review and meta-analysis. BMJ Open 2016;6:e012744.

9. Bohan A, Peter JB. Polymyositis and dermatomyositis (first of two parts). N Engl J Med 1975;292:344-7.

10. Bohan A, Peter JB. Polymyositis and dermatomyositis (second of two parts). N Engl J Med 1975;292:403-7.

11. Sontheimer RD. Would a new name hasten the acceptance of amyopathic dermatomyositis (dermatomyositis siné myositis) as a distinctive subset within the idiopathic inflammatory dermatomyopathies spectrum of clinical illness? J Am Acad Dermatol 2002;46:626-36.

12. Sontheimer RD, Miyagawa S. Potentially fatal interstitial lung disease can occur in clinically amyopathic dermatomyositis. J Am Acad Dermatol 2003;48:797-8.

13. Hayden JA, Côté $P$, Bombardier $C$. Evaluation of the quality of prognosis studies in systematic reviews. Ann Intern Med 2006;144:427-37.

14. Chen Z, Cao M, Plana MN, et al. Utility of anti-melanoma differentiation-associated gene 5 antibody measurement in identifying patients with dermatomyositis and a high risk for developing rapidly progressive interstitial lung disease: a review of the literature and a meta-analysis. Arthritis Care Res 2013;65:1316-24.

15. Riley RD, Higgins JP, Deeks JJ. Interpretation of random effects meta-analyses. BMJ 2011;342:d549.

16. Green S, Higgins JPT. Cochrane Handbook for Systematic Reviews of Interventions Version 5.1.0: The Cochrane Collaboration, 2011.

17. Egger M, Davey Smith G, Schneider M, et al. Bias in meta-analysis detected by a simple, graphical test. BMJ 1997;315:629-34.

18. Iorio A, Spencer FA, Falavigna M, et al. Use of GRADE for assessment of evidence about prognosis: rating confidence in estimates of event rates in broad categories of patients. BMJ 2015;350:h870.

19. Hozumi H, Fujisawa T, Enomoto $\mathrm{N}$, et al. Clinical utility of $\mathrm{YKL}-40$ in polymyositis/dermatomyositis-associated interstitial lung disease. $J$ Rheumatol 2017;44:1394-401.

20. Enomoto $\mathrm{Y}$, Suzuki Y, Hozumi H, et al. Clinical significance of soluble CD163 in polymyositis-related or dermatomyositis-related interstitial lung disease. Arthritis Res Ther 2017;19.;1:.9:9.

21. Hozumi H, Enomoto N, Kono M, et al. Prognostic significance of anti-aminoacyl-tRNA synthetase antibodies in polymyositis/ dermatomyositis-associated interstitial lung disease: a retrospective case control study. PLoS One 2015;10:e0120313.

22. Fujisawa T, Hozumi H, Kono M, et al. Prognostic factors for myositisassociated interstitial lung disease. PLoS One 2014;9:e98824.

23. Gono T, Sato S, Kawaguchi $\mathrm{Y}$, et al. Anti-MDA5 antibody, ferritin and IL-18 are useful for the evaluation of response to treatment in interstitial lung disease with anti-MDA5 antibody-positive dermatomyositis. Rheumatology 2012;51:1563-70.

24. Gono T, Kawaguchi Y, Satoh T, et al. Clinical manifestation and prognostic factor in anti-melanoma differentiation-associated gene 5 antibody-associated interstitial lung disease as a complication of dermatomyositis. Rheumatology 2010;49:1713-9.

25. Li T, Guo L, Chen Z, et al. Pirfenidone in patients with rapidly progressive interstitial lung disease associated with clinically amyopathic dermatomyositis. Sci Rep 2016;6:33226.

26. Zou J, Guo Q, Chi J, et al. HRCT score and serum ferritin level are factors associated to the 1-year mortality of acute interstitial lung disease in clinically amyopathic dermatomyositis patients. Clin Rheumatol 2015;34:707-14.

27. Ye S, Chen XX, Lu XY, et al. Adult clinically amyopathic dermatomyositis with rapid progressive interstitial lung disease: a retrospective cohort study. Clin Rheumatol 2007;26:1647-54.

28. Kurasawa K, Arai S, Namiki Y, et al. Tofacitinib for refractory interstitial lung diseases in anti-melanoma differentiation-associated 5 gene antibody-positive dermatomyositis. Rheumatology 2018:key188.

29. Sugiyama Y, Yoshimi R, Tamura M, et al. The predictive prognostic factors for polymyositis/dermatomyositis-associated interstitial lung disease. Arthritis Res Ther 2018;20:7.

30. Zhang T, Zhang J, Liu X, et al. A clinical analysis of prognostic factors for dermatomyositis-associated interstitial lung disease. Int J Clin Exp Med 2018;11:5903-11.

31. Okabayashi $\mathrm{H}$, Ichiyasu $\mathrm{H}$, Hirooka $\mathrm{S}$, et al. Clinical effects of direct hemoperfusion using a polymyxin B-immobilized fiber column in clinically amyopathic dermatomyositis-associated rapidly progressive interstitial pneumonias. BMC Pulm Med 2017;17:134.

32. Ikeda S, Arita M, Morita M, et al. Interstitial lung disease in clinically amyopathic dermatomyositis with and without anti-MDA-5 antibody: to lump or split? BMC Pulm Med 2015;15:159.

33. Rojas-Serrano J, Herrera-Bringas D, Mejía M, et al. Prognostic factors in a cohort of antisynthetase syndrome (ASS): serologic profile is associated with mortality in patients with interstitial lung disease (ILD). Clin Rheumatol 2015;34:1563-9.

34. Takada T, Aoki A, Asakawa K, et al. Serum cytokine profiles of patients with interstitial lung disease associated with antiCADM-140/MDA5 antibody positive amyopathic dermatomyositis. Respir Med 2015;109:1174-80.

35. Nara M, Komatsuda A, Omokawa A, et al. Serum interleukin 6 levels as a useful prognostic predictor of clinically amyopathic dermatomyositis with rapidly progressive interstitial lung disease. Mod Rheumatol 2014;24:633-6.

36. Chen $D$, Wang $X B$, Zhou $Y$, et al. Efficacy of infliximab in the treatment for dermatomyositis with acute interstitial pneumonia: a study of fourteen cases and literature review. Rheumatol Int 2013;33:2455-8.

37. Muro Y, Sugiura K, Akiyama M, et al. Limitations of a single-point evaluation of anti-MDA5 antibody, ferritin, and IL-18 in predicting the prognosis of interstitial lung disease with anti-MDA5 antibodypositive dermatomyositis. Clin Rheumatol 2013;32:395-8.

38. Sato S, Kuwana M, Fujita T, et al. Anti-CADM-140/MDA5 autoantibody titer correlates with disease activity and predicts disease outcome in patients with dermatomyositis and rapidly progressive interstitial lung disease. Mod Rheumatol 2013;23:496-502.

39. Shimojima Y, Ishii W, Matsuda M, et al. Coadministration of cyclosporin a with prednisolone in acute interstitial pneumonia complicating polymyositis/dermatomyositis. Clin Med Insights Arthritis Musculoskelet Disord 2012;5:43-52.

40. Sun $Y$, Liu $Y$, Yan B, et al. Interstitial lung disease in clinically amyopathic dermatomyositis (CADM) patients: a retrospective study of 41 Chinese Han patients. Rheumatol Int 2013;33:1295-302.

41. Tanizawa K, Handa T, Nakashima R, et al. The prognostic value of HRCT in myositis-associated interstitial lung disease. Respir Med 2013;107:745-52.

42. Yamasaki Y, Yamada H, Ohkubo M, et al. Longterm survival and associated risk factors in patients with adult-onset idiopathic inflammatory myopathies and amyopathic dermatomyositis: experience in a single institute in Japan. J Rheumatol 2011;38:1636-43.

43. Ji SY, Zeng FQ, Guo Q, et al. Predictive factors and unfavourable prognostic factors of interstitial lung disease in patients with polymyositis or dermatomyositis: a retrospective study. Chin Med J 2010;123:517-22.

44. Mukae $\mathrm{H}$, Ishimoto $\mathrm{H}$, Sakamoto $\mathrm{N}$, et al. Clinical differences between interstitial lung disease associated with clinically amyopathic dermatomyositis and classic dermatomyositis. Chest 2009;136:1341-7.

45. Hayashi S, Tanaka M, Kobayashi H, et al. High-resolution computed tomography characterization of interstitial lung diseases in polymyositis/dermatomyositis. J Rheumatol 2008;35:260-9.

46. Won Huh J, Soon Kim D, Keun Lee C, et al. Two distinct clinical types of interstitial lung disease associated with polymyositisdermatomyositis. Respir Med 2007;101:1761-9.

47. Kameda $\mathrm{H}$, Nagasawa $\mathrm{H}$, Ogawa $\mathrm{H}$, et al. Combination therapy with corticosteroids, cyclosporin A, and intravenous pulse cyclophosphamide for acute/subacute interstitial pneumonia in patients with dermatomyositis. J Rheumatol 2005;32:1719-26. 
48. Kang EH, Lee EB, Shin KC, et al. Interstitial lung disease in patients with polymyositis, dermatomyositis and amyopathic dermatomyositis. Rheumatology 2005;44:1282-6.

49. Ito M, Kaise S, Suzuki S, et al. Clinico-laboratory characteristics of patients with dermatomyositis accompanied by rapidly progressive interstitial lung disease. Clin Rheumatol 1999;18:462-7.

50. Tazelaar HD, Viggiano RW, Pickersgill J, et al. Interstitial lung disease in polymyositis and dermatomyositis. Clinical features and prognosis as correlated with histologic findings. Am Rev Respir Dis 1990;141:727-33.

51. Ichikado K, Suga M, Müller NL, et al. Acute interstitial pneumonia: comparison of high-resolution computed tomography findings between survivors and nonsurvivors. Am J Respir Crit Care Med 2002;165:1551-6.

52. Marie I, Hachulla E, Chérin $P$, et al. Interstitial lung disease in polymyositis and dermatomyositis. Arthritis Rheum 2002;47:614-22.

53. Lega JC, Fabien N, Reynaud Q, et al. The clinical phenotype associated with myositis-specific and associated autoantibodies: a meta-analysis revisiting the so-called antisynthetase syndrome. Autoimmun Rev 2014;13:883-91.

54. Cao H, Pan M, Kang Y, et al. Clinical manifestations of dermatomyositis and clinically amyopathic dermatomyositis patients with positive expression of anti-melanoma differentiation-associated gene 5 antibody. Arthritis Care Res 2012;64:1602-10.

55. Koga T, Fujikawa K, Horai $\mathrm{Y}$, et al. The diagnostic utility of antimelanoma differentiation-associated gene 5 antibody testing for predicting the prognosis of Japanese patients with DM. Rheumatology 2012;51:1278-84.

56. Gono T, Kawaguchi Y, Kuwana M, et al. Brief report: Association of HLA-DRB $1{ }^{*} 0101 /{ }^{*} 0405$ with susceptibility to antimelanoma differentiation-associated gene 5 antibody-positive dermatomyositis in the Japanese population. Arthritis Rheum 2012;64:3736-40.

57. Muro Y, Sugiura K, Hoshino K, et al. Epidemiologic study of clinically amyopathic dermatomyositis and anti-melanoma differentiationassociated gene 5 antibodies in central Japan. Arthritis Res Ther 2011;13:R214

58. Parronchi P, Radice A, Palterer B, et al. MDA5-positive dermatomyositis: an uncommon entity in Europe with variable clinical presentations. Clin Mol Allergy 2015;13:22.

59. Royston P, Altman DG, Sauerbrei W. Dichotomizing continuous predictors in multiple regression: a bad idea. Stat Med 2006;25:127-41. 